\title{
Análisis de la implementación de un ciclo de mejora docente en la asignatura Ciencia y Tecnología del Medio Ambiente
}

\author{
JUAN MANUEL MANCILLA LEYTÓN \\ Universidad de Sevilla \\ Departamento de Biología Vegetal y Ecología \\ jmancilla@us.es \\ ORCID: https://orcid.org/0000-0002-9042-6683 \\ D.O.I.: http://dx.doi.org/10.12795/JDU.2018.i01.77 \\ Pp.: 1376-1393
}

\section{Resumen}

El presente ciclo de mejora docente (CMD) ha sido realizado en la asignatura Ciencia y Tecnología del Medio Ambiente de 2o curso del Grado de Ingeniería Agrícola (Universidad de Sevilla). Los principales problemas detectados en esta asignatura, en los cursos previos a este CMD, han sido: escasa asistencia a clase, poca o nula asistencia a tutorías y aplazamiento del estudio de los contenidos a los días previos al examen final. Este CMD se ha centrado en las interacciones biológicas entre poblaciones y se ha llevado a cabo en 5 sesiones (4 teóricas y 1 práctica), durante las cuales se planificaron un conjunto de actividades basadas en ejemplos prácticos y dinámicas grupales que potenciaron la reflexión sobre los contenidos por parte de los estudiantes. La experiencia realizada ha sido muy gratificante y los resultados obtenidos indican una valoración muy positiva por parte de los estudiantes por este tipo de metodología docente. 
Palabras claves: Ciencia y Tecnología del Medio Ambiente, Grado en Ingeniería Agrícola, Docencia Universitaria, Experimentación Docente Universitaria, Interacciones Biológicas.

\section{Breve descripción del contexto}

Durante el primer cuatrimestre del curso académico 2018-2019 se ha realizado un ciclo de mejora docente (CMD) en la asignatura Ciencia y Tecnología del Medio Ambiente. Se trata de una asignatura obligatoria de 2 o curso del Grado de Ingeniería Agrícola de la Escuela Técnica Superior de Ingeniería Agronómica (Universidad de Sevilla). En esta asignatura se dan a conocer los principios ecológicos que gobiernan la Agricultura y sus implicaciones en el manejo agrícola y ganadero. Se tratan, pues, principios básicos que los estudiantes pueden aplicar, durante su etapa profesional, a situaciones específicas y problemas concretos. La escasa asistencia a clase y tutorías durante el curso así como dejar el estudio de la asignatura a los días previos al examen final, son los principales problemas detectados en la asignatura en los cursos académicos anteriores. Estos problemas se han traducido en una importante merma del rendimiento académico y por tanto un índice bajo de aprobados ( $<30 \%$ de los estudiantes matriculados).

En el presente curso académico, el grupo en el que se desarrolla el CMD consta de un total de 59 estudiantes matriculados, de los cuales 18 son repetidores. Para el desarrollo de la asignatura la asistencia a las sesiones teóricas no es obligatoria, y hasta la fecha se sitúa en torno al 70\% (40 estudiantes). Las sesiones de clases teóricas se desarrollan en 55 minutos (lunes 10:50-11:45h y jueves 13:3514:30h) y las sesiones de prácticas en 1 hora y 50 minutos (grupos de 20 alumnos máximo; martes y miércoles en distintos horarios). 


\section{Diseño previo del Ciclo de Mejora Docente}

El presente CMD se ha centrado en las interacciones biológicas entre poblaciones. Durante las distintas sesiones que se contemplan en el CDM, mi objetivo ha sido que los estudiantes comprendan que la biomasa de las poblaciones tanto de consumidores como de productores primarios depende de las interacciones que tengan no sólo con el medio que los rodea (biotopo), sino que también depende de las que mantengan con el resto de las especies que componen el ecosistema (biocenosis), ya que como se verá en esta parte de la asignatura tanto la competencia como la predación pueden reducir la biomasa del nivel trófico inferior. No obstante, no es la única interacción que se produce en estos sistemas, sino que existen otras muchas interacciones, las cuales pueden tener un efecto muy distinto (p.e. Mutualismo, Amensalismo, Comensalismo, etc.).

Los contenidos a desarrollar, durante este CMD, quedan recogidos de manera esquemática en la Figura 1, y son se muestran, in extenso, a continuación:

1. Contenidos teóricos: tipos de relaciones (directas e indirectas); tipos de interacciones entre especies (competencia, predación, mutualismo, parasitoidismo y otras relaciones); conocimiento de las relaciones entre poblaciones en los agroecosistemas; conocimiento de las interacciones entre el hombre y las especies domésticas; y conocimiento de las interacciones entre especies domésticas y entre poblaciones domésticas y silvestres.

2. Contenidos procedimentales: utilización y manejo del flujo de energía a través las cadenas y redes tróficas a favor de los sistemas agroecológicos; cuantificación del flujo de energía a través de los distintos consumidores y sus interacciones (cadena y red trófica); y aplicabilidad, uso y 
direccionalidad de las interacciones biológicas a favor de los sistemas agroecológicos (control y gestión de plagas; control Biológico).

3. Contenidos actitudinales: compromiso afectivo con el medio que les rodea; y preocupación y respeto medioambiental.

Los contenidos quedaron estructuraron en tres bloques: i) Bases ecológicas de las interacciones intra e interespecíficas (respondería a las preguntas claves ¿Cuáles son? ¿Cómo son?), ii) Interacciones entre ecosistemas, agroecosistemas y actividades agropecuarias (respondería a las preguntas ¿Dónde ocurren? ¿Entre quienes se dan?), y iii) Control y gestión de las interacciones (respondería a las preguntas ¿Quién las gestiona? ¿De qué nos beneficiamos?) (Figura 1).

\section{INTERACCIONES ENTRE POBLACIONES}

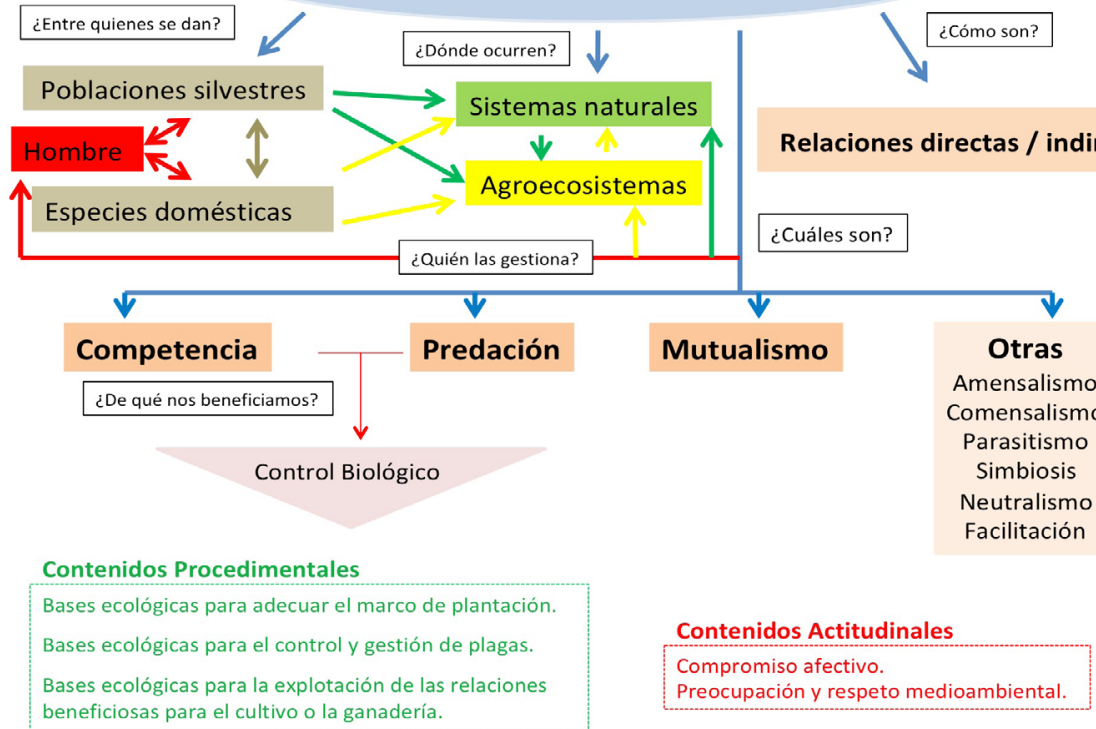

Figura 1. Mapa de contenidos a desarrollar durante del CMD

Con respecto a mi modelo metodológico, éste ha experimentado una gran evolución y sufrido algunos cambios importantes durante el desarrollo del Curso General de 
Docencia Universitaria realizado dentro del Programa de Formación e Innovación Docente del Profesorado de la Universidad de Sevilla. A continuación se detalla el modelo metodológico aplicado en el presente CMD. En cada inicio de sesión contemplo una recapitulación (Rp) de lo que se ha impartido en las sesiones anteriores y suelo dedicar unos 5-10 minutos para resolver dudas o cuestiones que hayan quedado pendientes. Durante el desarrollo de este CDM he priorizado el trabajo en clase a través de ejemplos o problemas reales $(P)$, los cuales han sido alternados con los conceptos teóricos a desarrollar (T), lo que ha permito crear un ambiente crítico natural (Bain, 2007), en el que los estudiantes estuvieran más predispuesto a un aprendizaje más atractivo y dinámico. Además, durante las sesiones, he dado cabida a un tiempo de reflexión / discusión (R/D) por parte de los estudiantes, de manera que he dejado de tener yo el papel protagonista en la clase. Por último, al final de cada sesión he reservado un pequeño intervalo de tiempo (5-10 minutos) destinado a resaltar las conclusiones finales y cierre de la sesión. En este CMD incorporo, por primera vez, la exploración de las ideas de los estudiantes al inicio (Ip1) y al final (Ip2) del desarrollo de los contenidos para poder realizar la evaluación del proceso. En la Figura 2 queda recogido el modelo metodológico descrito y aplicado en el presente CMD.

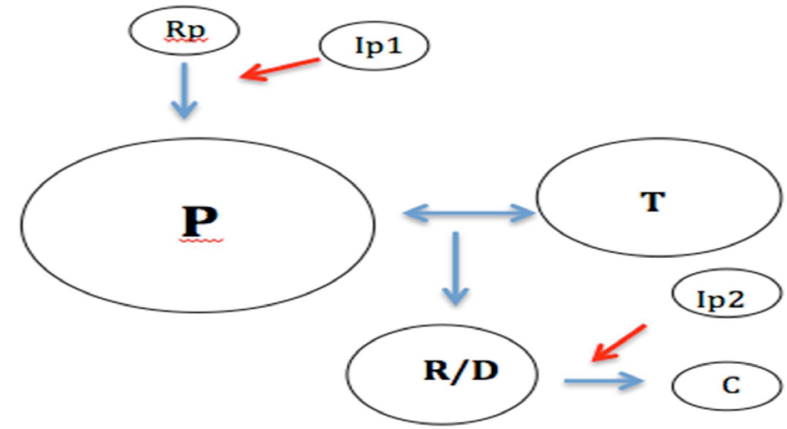

Figura 2. Modelo metodológico aplicado en el CMD. Recapitulación (Rp); Exploración de Ideas inicial (Ip1) y final (Ip2); Contenidos teóricos (T); Problemas o ejemplos (P); Reflexión / Discusión conjunta profesor-alumno; Cierre de sesión y Conclusiones (C).

Jornadas de Formación e Innovación Docente del Profesorado | № 1 (2018) Esta obra se distribuye con la licencia Creative Commons 


\section{Secuencia de actividades programada}

El CMD se ha desarrollado en 4 sesiones teóricas y 1 sesión práctica durante las cuales se han impartido los contenidos descritos anteriormente. Todos los contenidos conceptuales quedan recogido en el manual Ecología para la Agricultura, protocolos de prácticas y lecturas recomendadas. Todo este material ha sido desarrollado por los profesores que imparten o han impartido la asignatura. El material complementario necesario para el desarrollo del CMD le ha sido suministrado a los estudiantes en clase o a través de la plataforma Web-CT. En la tabla 1 se detalla la secuencia de actividades que se ha desarrollo durante el presente CDM.

Tabla 1. Descripción de las distintas actividades que constituyen las distintas sesiones contempladas en el Ciclo de Mejora Docente.

Jornadas de Formación e Innovación Docente del Profesorado | № 1 (2018) Esta obra se distribuye con la licencia Creative Commons 


\begin{tabular}{|c|c|c|}
\hline & ACTIVIDADES & MODELO \\
\hline \multirow{6}{*}{ 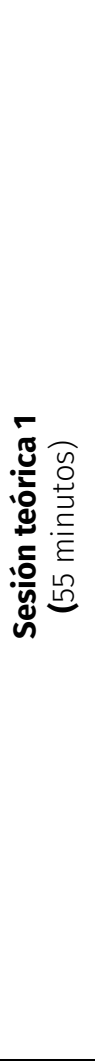 } & $\begin{array}{l}\text { Actividad 1. Recapitulación (Rp). Al igual que en } \\
\text { todas las sesiones previas se comienza con un } \\
\text { pequeño repaso de los contenidos impartidos } \\
\text { anteriormente y la resolución de cuestiones o } \\
\text { dudas pendientes (5-10 minutos). }\end{array}$ & \\
\hline & $\begin{array}{l}\text { Actividad 2. Exploración de las ideas previas } \\
\text { de los estudiantes sobre los contenidos que } \\
\text { se van a impartir en este CMD. El cuestionario } \\
\text { está constituido por } 4 \text { preguntas abiertas. Ten- } \\
\text { drán un tiempo máximo para contestarla de } 10 \\
\text { minutos. }\end{array}$ & \\
\hline & $\begin{array}{l}\text { Actividad 3. Visualización del video How Wolves } \\
\text { Change Rivers. } \\
\text { https://www.youtube.com/watch?v=ysa50- } \\
\text { BhXz-Q. (5 minutos) }\end{array}$ & \\
\hline & $\begin{array}{l}\text { Actividad 4. Trabajo en grupos de } 4 \text { sobre un } \\
\text { ejemplo similar de control trófico en ecosiste- } \\
\text { mas acuáticos ( } 10 \text { minutos). }\end{array}$ & \\
\hline & $\begin{array}{l}\text { Actividad 5. Visualización del video How Whales } \\
\text { Change Climate https://www.youtube.com/wat- } \\
\text { ch?v=M18HxXve3CM (5 minutos). }\end{array}$ & \\
\hline & $\begin{array}{l}\text { Actividad 6. Discusión por parte del profesor y } \\
\text { los estudiantes de las relaciones tróficas e inte- } \\
\text { racciones que se producen en los ecosistemas } \\
\text { (15 minutos). }\end{array}$ & \\
\hline \multirow{4}{*}{ 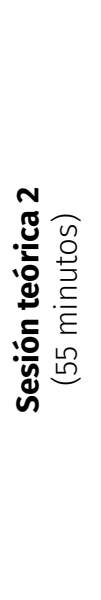 } & Actividad 1.Recapitulación (Rp) (5-10 minutos). & \multirow{4}{*}{ RP } \\
\hline & $\begin{array}{l}\text { Actividad 2. Exposición por el profesor de las } \\
\text { similitudes y diferencias entre los ecosiste- } \\
\text { mas naturales y sistemas agropecuarios (10 } \\
\text { minutos). }\end{array}$ & \\
\hline & $\begin{array}{l}\text { Actividad 3. Trabajo en grupos de } 4 \text { sobre las } \\
\text { perturbaciones que se producen en los siste- } \\
\text { mas agropecuarios y sus implicaciones en los } \\
\text { ecosistemas naturales ( } 20 \text { minutos). }\end{array}$ & \\
\hline & $\begin{array}{l}\text { Actividad 4. Discusión por parte del profesor y } \\
\text { los alumnos/as de las relaciones tróficas e in- } \\
\text { teracciones que se producen entre los ecosis- } \\
\text { temas naturales y sistemas agropecuarios (15 } \\
\text { minutos). }\end{array}$ & \\
\hline
\end{tabular}

Jornadas de Formación e Innovación Docente del Profesorado | № 1 (2018) Esta obra se distribuye con la licencia Creative Commons Reconocimiento-NoComercial-SinObraDerivada Internacional (CC BY-NC-ND 4.0.) 


\begin{tabular}{|c|c|c|}
\hline \multirow{5}{*}{ 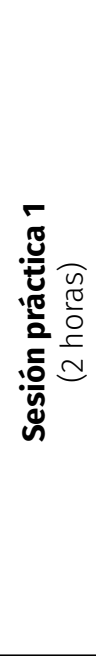 } & Actividad 1.Recapitulación (Rp) (5 minutos). & \multirow{5}{*}{$\frac{1}{p}$} \\
\hline & $\begin{array}{l}\text { Actividad 2. Explicación por parte del profesor } \\
\text { el Estudio del Impacto Ambiental de las acti- } \\
\text { vidades agropecuarias. Procedimiento, técni- } \\
\text { cas y desarrollo del Inventario Ambiental ( } 30 \\
\text { minutos). }\end{array}$ & \\
\hline & $\begin{array}{l}\text { Actividad 3. Exposición y presentación de un } \\
\text { caso real por parte del profesor (10 minutos). }\end{array}$ & \\
\hline & $\begin{array}{l}\text { Actividad 4. Trabajo en grupos de } 4 \text { para elabo- } \\
\text { rar la metodología a seguir en el desarrollo del } \\
\text { Inventario Ambiental del ejemplo expuesto ( } 60 \\
\text { minutos). }\end{array}$ & \\
\hline & $\begin{array}{l}\text { Actividad 5. Discusión y conclusiones finales de } \\
\text { la importancia del Inventario Ambiental en el } \\
\text { conocimiento de las interacciones (15 minutos). }\end{array}$ & \\
\hline \multirow{3}{*}{ 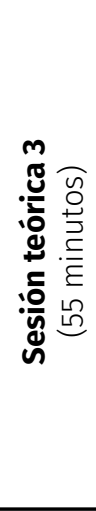 } & Actividad 1. Recapitulación (Rp) (5 minutos). & \multirow{6}{*}{$\begin{array}{l}\text { El modelo se completa } \\
\text { entre las } 2 \text { sesiones }\end{array}$} \\
\hline & $\begin{array}{l}\text { Actividad 2. Presentación por el profesor del } \\
\text { trabajo a realizar durante la sesión. Se le facili- } \\
\text { tará distintas fotografías y preguntas relaciona- } \\
\text { das con los distintos tipos de Interacciones (10 } \\
\text { minutos). }\end{array}$ & \\
\hline & $\begin{array}{l}\text { Actividad 3. Trabajo en grupos de } 4 \text { para tra- } \\
\text { bajar y discutir las fotografías y preguntas pro- } \\
\text { puestas ( } 40 \text { minutos). Si no tienen tiempo } \\
\text { suficiente para acabar la actividad deberán } \\
\text { terminarla en casa, se discute en la próxima } \\
\text { sesión. }\end{array}$ & \\
\hline \multirow{3}{*}{ 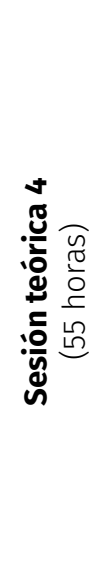 } & $\begin{array}{l}\text { Actividad 1. Discusión por parte del profesor y } \\
\text { los estudiantes de la actividad de la sesión an- } \\
\text { terior ( } 30 \text { minutos). }\end{array}$ & \\
\hline & $\begin{array}{l}\text { Actividad 2. Exposición / Conclusión por el pro- } \\
\text { fesor de las implicaciones e importancia de las } \\
\text { interacciones biológicas para la Ingeniería ( } 10 \\
\text { minutos). }\end{array}$ & \\
\hline & $\begin{array}{l}\text { Actividad 3. Exploración final de las ideas de } \\
\text { los estudiantes sobre los contenidos que se } \\
\text { han impartido en este CMD. Tendrán que res- } \\
\text { ponder a las mismas preguntas que se les } \\
\text { dio al inicio del CMD. En este caso se am- } \\
\text { plió el tiempo para contestar las preguntas ( } 15 \\
\text { minutos). }\end{array}$ & \\
\hline
\end{tabular}

Jornadas de Formación e Innovación Docente del Profesorado | № 1 (2018) Esta obra se distribuye con la licencia Creative Commons Reconocimiento-NoComercial-SinObraDerivada $\quad 4.0$ Internacional (CC BY-NC-ND 4.0.) 


\section{Aplicación del CMD}

\section{Relato resumido de las sesiones}

Sesión teórica 1; uno de mis propósitos para este CMD fue realizar una adecuada gestión del tiempo. Antes de cada sesión, establecí distintas alarmas en mi teléfono móvil para controlar los intervalos de trabajo. Tras hacer una pequeña recapitulación de la sesión anterior les pasé a los estudiantes el cuestionario inicial. Tras recoger los cuestionarios, como tenía previsto, les puse el video How Wolves Change Rivers. El contenido del video trata sobre cambios en las redes tróficas en ecosistemas terrestres. Durante el desarrollo del mismo todos estuvieron en silencio y atentos. Tras la proyección del video, tal y como tenía fijado, trabajaron en grupos sobre un caso real. Me fui paseando de un grupo a otro escuchando lo que decían, sin decirles nada. A un grupo tuve que dedicarle unos minutos porque no acababan de entender lo que les pedía y volví a explicarles la actividad. Otro dos grupos, cuando me acerqué, dejaron de debatir entre ellos para contarme a mí lo que estaban discutiendo, y los "ignoré" un poco, les dije que luego tendríamos tiempo para discutirlo entre todos y que siguieran trabajando. En general, la dinámica fue muy buena. A continuación, antes de discutir la actividad con ellos, les puse el video How Whales Change Climate. Es similar al anterior pero en este caso los cambios de la red trófica tiene lugar en el ecosistema acuático, al igual que en el ejemplo que ellos habían trabajado. De nuevo, todos estuvieron en silencio y atentos. Llegados a este momento, les dije que un representante de cada grupo fuera exponiendo las conclusiones a las que habían llegado en la actividad 4. Dos alumnos de grupos distintos pidieron la palabra. Tras la exposición del primero, di la palabra al segundo, el cual coincidió en varios aspectos del primero y aportó algunas cosas nuevas. Posteriormente intervinieron 
5 alumnos más, en la mayoría de los casos apoyando las primeras intervenciones. Tras esta exposición les proyecté un par de diapositivas sobre las relaciones tróficas e introduje algunos conceptos sobre tipos de interacciones biológicas que se iban a tratar en la próxima sesión. Durante esta sesión, gracias a las distintas alarmas del teléfono, fui capaz de cumplir con los intervalos de tiempo previstos. Los estudiantes mostraron gran interés por las actividades desarrolladas, alguno de ellos me lo manifestaron de manera explícita al finalizar las sesiones. Esto último me motivó mucho para continuar con el ciclo.

Sesión teórica 2; tras una pequeña recapitulación y tiempo de resolución de dudas les ofrecí algunas píldoras teóricas sobre las similitudes y diferencias entre los ecosistemas naturales y sistemas agropecuarios. Tras mi exposición les pedí que, en grupos de 4, seleccionaran una actividad agropecuaria (agrícola o ganadera) e identificaran las perturbaciones e implicaciones que esta actividad podría provocar en la red trófica de los ecosistemas naturales.

Al igual que en la sesión anterior, me fui paseando de un grupo a otro escuchando lo que decían, en una primera ronda sin decirles nada, y en una segunda animando y preguntando a aquellos grupos que no estaban trabajando para que lo hicieran. Tras esto, la dinámica fue similar a la sesión anterior. En esta ocasión participaron todos los grupos de trabajo en la discusión. Me ayudé de la pizarra para reforzar los resultados que fueron exponiendo y fui introduciendo, a la vez, diferentes píldoras teóricas para reforzar las ideas.

Sesión práctica 1; después de un pequeño repaso de la sesión práctica de la semana anterior, desarrollé los contenidos teóricos a impartir en esta sesión práctica. Esta práctica está muy relacionada con el bloque temático teórico sobre interacciones biológicas, pues el análisis ambiental 
de cualquier estudio de calidad requiere del conocimiento de las diferentes interacciones que se desarrollan o pueden desarrollarse en los distintos ecosistemas naturales y sistemas agropecuarios. Durante la explicación fue interrumpido en varias ocasiones por distintos estudiantes, por lo que para el desarrollo de mi intervención necesito un poco más de tiempo del que tenía previsto. Esto hizo que tuviera que reducir el tiempo que había pensado dedicar al análisis en grupo del caso de estudio. La dinámica de trabajo fue similar a las anteriores; aunque en más de un grupo de trabajo tuve que redirigir la actividad ya que no estaban trabajando. Tras el trabajo en grupo, debatimos los resultados a los que los estudiantes habían llegado, aunque no al nivel que tenía previsto ya que el retraso acumulado de la actividad anterior no lo permitió. Por ello, tuve que añadir algunos instrumentos que no salieron durante el trabajo y posteriormente en la exposición, algo que no tenía previsto. Hubiera estado bien disponer de todo el tiempo de trabajo que tenía establecido (se trata de una de las cosas a mejorar en el futuro).

Sesión teórica 3; tras la resolución de algunas dudas surgidas, les expliqué la actividad programada para esta sesión. Disponían de un total de 12 fotografías / imágenes, cada una con una pregunta, las cuales estaban relacionadas con los distintos tipos de interacciones biológicas. Intenté aplicar la metodología vista en el Curos de Innovación "dar clases con la boca cerrada" (Finkel, 2008), así que durante el resto de la sesión me paseé por cada grupo y me paré unos minutos sólo para escuchar lo que estaban discutiendo. Evité responderles preguntas directas porque la discusión de las preguntas las quería dejar para la siguiente sesión. La mayoría de los grupos fueron capaces de terminar la actividad en el tiempo que tenía previsto, aunque algún grupo tuvo que acabar la actividad en casa. 
Sesión teórica 4; la mayor parte de esta sesión la dedicamos a discutir los resultados de la sesión anterior. Grupo a grupo fueron desarrollando y justificando sus respuestas. A través de las distintas intervenciones pude percibir, en la mayoría de los estudiantes, una gran implicación por el trabajo propuesto. Tras sus exposiciones hice hincapié en los aspectos más importantes, y los reforcé anotando las principales ideas en la pizarra. De nuevo, aunque intenté controlar el tiempo, se alargó mucho más de lo tenía previsto, por lo que no pude pasar el cuestionario final en esta sesión. El cuestionario final lo realizaron en la siguiente sesión teórica, fuera de las sesiones planificadas para el CMD.

\section{Evaluación de los modelos iniciales y finales de los estudiantes}

A continuación se realiza el análisis comparativo de las respuestas obtenidas al cuestionario inicial y final, según la metodología descrita (Porlán et al., 2017). Para este análisis, sólo se han considerado a un total de 19 estudiantes, ya que fueron los únicos que completaron el CMD, es decir, que realizaron todas las actividades programadas y ambos cuestionarios. No se incluyen los resultados parciales de otros estudiantes para evitar malinterpretaciones de los mismos. Las preguntas fueron las mismas en el cuestionario inicial y final.

\section{Pregunta 1.- Describe brevemente una actividad agri- cola sobre la que tengas experiencia o que conozcas.}

En realidad esta pregunta no estaba pensada para obtener información sobre los contenidos a tratar en este CMD. Sólo se trataba de situar al estudiante y hacer que el resto de las preguntas fueran específicas de esa actividad. Es por ello que no se incluye su análisis. 


\section{Pregunta 2.- ¿Qué plagas pueden afectar a la actividad que has descrito?}

Esta pregunta buscaba explorar el nivel de conocimiento que tiene los estudiantes sobre las plagas que pueden afectar a las actividades agrícolas. Las distintas categorías encontradas en las respuestas de los estudiantes se detallan a continuación: A.- Cita ejemplos con terminología apropiada y describe relaciones; B.- Cita ejemplos con vocabulario no específico; C.- Cita ejemplos pero no están relacionados con la actividad descrita; y iv) D.- No cita ejemplos / Interpreta la respuesta libremente y no se ciñe a la pregunta (Figura 3).

\section{$(11 \%)$ A $(42 \%)$}

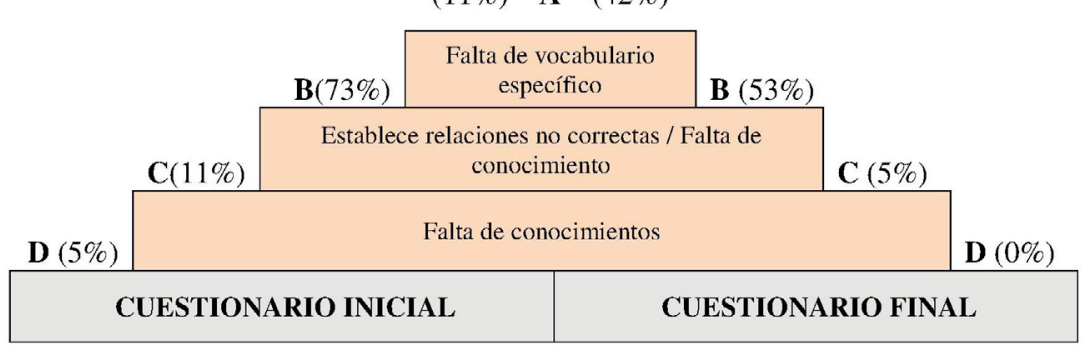

Figura 3. Respuestas a la pregunta 2. Junto a cada categoría se acompaña el porcentaje de estudiantes con dicha respuesta. En la base se encuentra la respuesta más simple (D) y en el ápice la respuesta de mayor complejidad

(A). Debajo de cada escalón se detalla el obstáculo.

Las respuestas a la pregunta 2, durante el cuestionario inicial, pusieron de manifiesto la falta de conocimientos específicos de los estudiantes sobre los conceptos y contenidos a tratar. El análisis de las respuestas del cuestionario final muestra una evolución de casi la mitad de los estudiantes hacia una categoría de respuesta más completa y compleja que la desarrollada en el cuestionario inicial. 


\section{Pregunta 3.-_Conoces las relaciones que se dan entre la actividad y las plagas descritas? En caso afirmativo de- talla la respuesta.}

Con esta pregunta se indagaba en el conocimiento de las interacciones biológicas entre poblaciones. La mayoría de los estudiantes, excepto 4, contestaron afirmativamente la pregunta. Las respuestas detalladas han constituido las siguientes categorías: A.- Describe interacciones biológicas; B.- Reconoce las interacciones biológicas pero no usa vocabulario específico; C.- Relación negativa (respuesta básica); y D.- No sabe / no contesta (Figura 4).

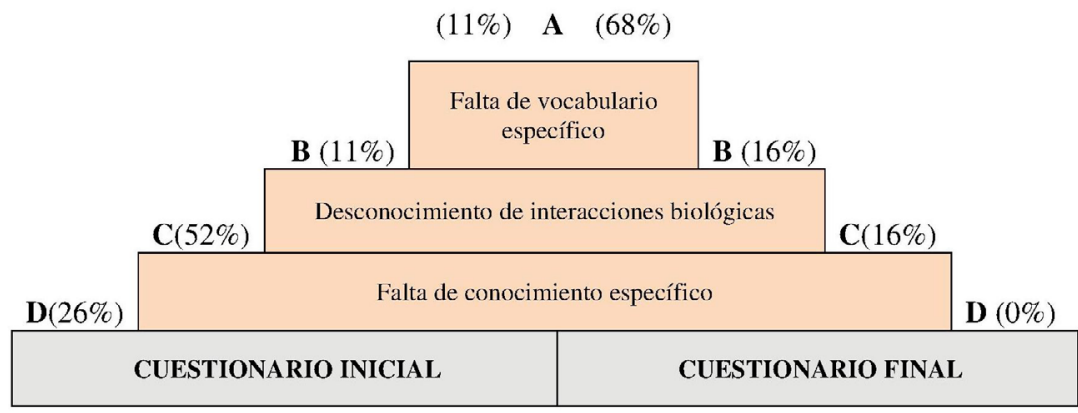

Figura 4. Respuestas a la pregunta 3. Junto a cada categoría se acompaña el porcentaje de estudiantes con dicha respuesta. En la base se encuentra la respuesta más simple (D) y en el ápice la respuesta de mayor complejidad

(A). Debajo de cada escalón se detalla el obstáculo.

Al igual que la pregunta anterior, en esta pregunta, tras la impartición de este CMD, la mayoría de los estudiantes evolucionaron en su modelo. Las interacciones biológicas han sido el eje central de los contenidos impartido en este CMD, es por ello que esta evolución era esperada. Hay que indicar que la respuesta a esta pregunta es muy específica, debido a la actividad seleccionada por cada estudiante, y puede ser por eso que la evolución del modelo es algo menor de lo esperado. 


\section{Pregunta 4.- ¿Crees que es posible evitar la propaga- ción de las plagas en la actividad que has descrito? En caso afirmativo, ¿qué métodos emplearías?}

Con esta pregunta se exploró el conocimiento de las interacciones biológicas en los ecosistemas y por tanto de los distintos métodos de control de las plagas. Todos los estudiantes afirmaron que se podría evitar la propagación. No obstante, las metodologías detalladas que emplearían fueron diversas. A continuación se detallan las distintas categorías obtenidas: A.- Lucha integrada; B.- Lucha biológica; C.- Lucha química y/o física-mecánica; y D.- Afirma que se puede evitar pero no detalla los métodos (Figura 5).

\section{$(5 \%)$ A $(79 \%)$}

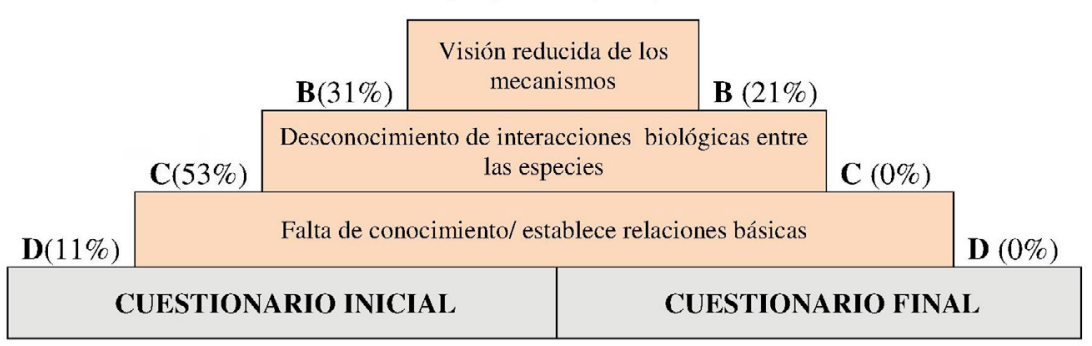

Figura 5. Respuestas a la pregunta 4. Junto a cada categoría se acompaña el porcentaje de estudiantes con dicha respuesta. En la base se encuentra la respuesta más simple (D) y en el ápice la respuesta de mayor complejidad

(A). Debajo de cada escalón se detalla el obstáculo.

Tras la impartición del presente CMD, se puede comprobar una gran evolución del modelo de los estudiantes hacía las respuestas más completas y complejas ( $\mathrm{A}$ y B) en el cuestionario final, desapareciendo, en la pregunta 4, las respuestas más simples (categoría D). Todos los estudiantes, excepto 1, evolucionaron a una categoría o clase superior. Tal vez esta gran evolución estuvo motivada porque los contenidos de esta pregunta fueron impartidos al final del CMD, por lo que los estudiantes los tenían más recientes. 


\section{Evaluación del CMD}

\section{Cuestiones a mantener y cambios a introducir para el futuro}

Las principales dificultades encontradas durante el desarrollo de este CMD se recogen a continuación:

- Gestión del tiempo; aunque desde el inicio me propuse controlar los distintos tiempos invertidos en las actividades programadas, incluso ayudándome de la tecnología móvil, no he sido capaz de cumplir con todos los intervalos previstos, por lo que en más de una sesión tuve que rectificar sobre la marcha y reducir el tiempo de las actividades planteadas al final de las sesiones. Peco de ser ambicioso y no realista; en el diseño de las próximas actividades dejaré un margen de tiempo para posibles imprevistos, de manera que no se vea mermado en ningún momento el tiempo para el desarrollo de las mismas.

- Mantener el nivel de atención e implicación de los estudiantes durante todo el CMD. Fundamentalmente en la asistencia a las últimas sesiones del Ciclo. Esto ha sido motivado, principalmente, por los exámenes parciales en otras asignaturas. En este caso no sé cuál sería la solución, ya que la asistencia a las sesiones teóricas de esta asignatura no son obligatorias.

- Incertidumbre de no mantener la dinámica de enseñanza-aprendizaje durante este CMD para el resto de sesiones del curso académico. Por mi corta experiencia en el desarrollo de innovación docente, puede ser difícil mantener esta metodología siempre, ya que los ritmos de impartición son muy distintos y en algún que otro contenido teórico es difícil de aplicar. No obstante mi intención es hacerlo cada vez que pueda, compartiendo con ellos problemas y casos prácticos o 
facilitando lecturas recomendadas fuera de las sesiones en clase.

- Mejorar el diseño de las actividades. Algunas actividades propuestas necesitan un re-diseño (p.e. actividad 4 de la sesión práctica) para redirigirla lo máximo al contenido y facilitar el trabajo en la clase. Así mismo, la actividad 3 de la sesión 3 teórica tengo que simplificarla para que de tiempo de ejecutarla durante la sesión, ya que con la experiencia de este año he podido comprobar que son pocos los que la acabaron fuera de clase.

\section{Experiencia a incorporar a toda la práctica docente habitual}

La sensación con la que me quedo después de la ejecución de este CMD es positiva. Aunque el desarrollo y preparación de este ciclo me ha supuesto mucho esfuerzo y trabajo, ha sido gratificante no sólo para mí, sino que también para mis estudiantes ha sido una experiencia enriquecedora en comparación con la tradicional clase magistral. La utilización de problemas reales y videos didácticos, así como el trabajo/discusión en grupo, ha permitido una mayor participación y protagonismo de los estudiantes durante las sesiones. Con este tipo de modelo se deja a un lado la clase magistral y se gana en protagonismo de los estudiantes, lo que repercute beneficiosamente en su implicación y aprendizaje (Finkel, 2008). La acogida, por parte de los estudiantes, de la metodología de trabajo planteada en este CMD fue muy buena y positiva, mostrando gran interés en la misma.

Por último me ha resultado muy interesante y útil el Diario de clase, aunque no en su versión detalla y extensa, sino en una versión más simple. Desde hace unas semanas, después de cada sesión dedico unos minutos a realizar pequeñas anotaciones donde resalto las cosas a mantener y sustituir para el próximo curso. 


\section{Principios didácticos del CMD y aplicación en el futuro}

Los principios didácticos que me gustaría mantener y aplicar, no sólo en esta asignatura sino el resto de asignaturas que imparto, quedan recogidos a continuación: i) con respecto a la metodología: "Es necesario diseñar / rediseñar nuestro modelo metodológico en función de las características de los contenidos a impartir"; ii) con respecto a los contenidos: "Los contenidos deben ser clasificados en conceptuales, procedimentales y actitudinales; jererquizarlos según la importancia de los mismos y establecer relaciones bidireccionales entre ellos (verticales y horizontales)"; iii) con respecto al aprendizaje de los estudiantes: "Es necesario explorar los modelos mentales de nuestros estudiantes para poder adaptar la metodología y profundidad de los contenidos en cada momento a los mismos", y iv) con respecto a la evaluación: "Es necesaria una evaluación inicial y final que nos permita analizar la evolución y consecución de los contenidos impartidos".

\section{Referencias bibliográficas}

Bain, K. (2007). Lo que hacen los mejores profesores universitarios. (2a edición). Valencia: Universitat de València.

Finkel, D. (2008). Dar clase con la boca cerrada. Valencia: Universitat de València.

Porlán, R. (Coord.) (2017). Enseñanza Universitaria. Cómo mejorarla. Madrid: Ediciones Morata. 